\title{
CÁlCUlo NumÉRico DE laS PROPIEDADES TÉRMICAS EFECTIVAS DE UN COMPUESTO POR ELEMENTOS FINITOS
}

\author{
NumERICAL CALCULATION OF THE \\ EFFECTIVE THERMAL PROPERTIES OF A \\ COMPOSITE BY FINITE ELEMENTS
}

\author{
Rodney Hechavarría ${ }^{1, *}$, Gonzalo López ${ }^{1}$, Francisco Pazmiño ${ }^{1}$, Maritza Ureña ${ }^{1}$, \\ Andrés Hidalgo ${ }^{1}$
}

\section{Resumen}

El desarrollo de nuevos métodos para determinar las propiedades térmicas de materiales compuestos está siempre en constante avance. En este estudio se propone un método unidimensional para el cálculo numérico de la conductividad y difusividad térmicas efectivas en materiales sólidos heterogéneos (compuestos), entre $\left[10-20{ }^{\circ} \mathrm{C}\right]$, mediante el uso del programa Solidworks® 2016, el cual se basa en el método de cálculo de elementos finitos. Primero, se obtiene la distribución de temperatura en función de la coordenada y el tiempo; luego, se ajusta el modelo teórico, la ecuación parabólica de difusión del calor en una dimensión, a los datos obtenidos en la simulación para obtener la solución. Inicialmente, se modela la distribución de temperatura en una barra sólida de cobre homogénea, material conocido, bajo un flujo de calor constante en $x=L$, arrojando valores de conductividad y difusividad térmicas de acuerdo con los reportados en la literatura, con un error relativo de $0,01 \%$ y $0,7 \%$ respectivamente.

\section{Abstract}

The development of new methods for determining the thermal properties of composite materials is always in constant progress. This study proposes a one-dimensional method for the numerical calculation of effective thermal conductivity and diffusivity in heterogeneous solid materials (composite), between $\left[10-20{ }^{\circ} \mathrm{C}\right]$, using the program Solidworks ${ }^{\circledR} \quad 2016$, which is based on the method of Finite Element Calculation. First, the temperature distribution is obtained as a function of the coordinate and time; then, the theoretical model, the Parabolic Heat Diffusion Equation in one dimension, is adjusted to the data obtained in the simulation to obtain the solution. Initially, the temperature distribution in a homogeneous solid copper bar, known material, is modeled under a constant heat flux at $x=L$, yielding thermal conductivity and diffusivity values in accordance with those reported in the literature, with a relative error of $0.01 \%$ and $0.7 \%$ respectively.

\footnotetext{
${ }^{1, *}$ Facultad de Ingeniería Civil y Mecánica, Universidad Técnica de Ambato, Ecuador. Autor para correspondencia : ro.hechavarria@uta.edu.ec. (D) http://orcid.org/0000-0002-0195-6157,

(D) http://orcid.org/0000-0003-4387-6216, (D) http://orcid.org/0000-0002-6530-043X,

(D) http://orcid.org/0000-0002-0667-5581, (D) http://orcid.org/0000-0001-5179-2405
}

Recibido: 21-11-2016, aprobado tras revisión: 05-05-2017

Forma sugerida de citación: Hechavarría, R.; López, G.; Pazmiño, F.; Ureña, M.; Hidalgo, A. (2017). «Cálculo numérico de las propiedades térmicas efectivas de un compuesto por elementos finitos». InGENIUs. N. ${ }^{\circ} 18$, (julio-diciembre). pp. 14-20. ISSN: 1390-650X. 
Luego se modela la distribución de temperatura en una barra sólida heterogénea a base de cobre (65,7 \%)-plomo (34,3\%) y, bajo la misma condición de flujo de calor, se obtienen los valores simulados de temperatura en función del tiempo con los cuales se calculan la conductividad y difusividad térmicas efectivas de este compuesto. Los resultados obtenidos muestran consistencia y confiabilidad pues se encuentran dentro del rango establecido por estudios anteriores.

Palabras clave: conductividad térmica, difusividad térmica, propiedades térmicas, compuesto, simulación, elementos finitos.
Then, the temperature distribution is modeled in a heterogeneous solid bar based on copper (65.7\%)lead $(34.3 \%)$ and, under the same heat flow condition, the simulated values of temperature in function of the time with which the effective thermal conductivity and diffusivity of this compound are calculated. The obtained results show consistency and reliability because they are within the range established by previous studies.

Keywords: thermal conductivity, thermal diffusivity, thermal properties, composite, simulation, finite elements. 


\section{Introducción}

Desde hace años existe el interés en el desarrollo de nuevos métodos para determinar la conductividad y difusividad térmicas de los compuestos. Esto se debe, en gran medida, al rápido avance de la ciencia de materiales y sus múltiples aplicaciones [1-14]. Existen los métodos estacionarios que, sin embargo, presentan la desventaja del prolongado tiempo de exposición al calor que sufre la muestra, aumentando considerablemente su temperatura y, por lo tanto, pudiendo incidir en la variación de sus características térmicas [6], [15], [16].

En los materiales heterogéneos es más compleja la determinación de los valores exactos de los parámetros térmicos por su composición [17-25]. Los materiales compuestos pueden ser representados por medios laminados, fibrosos, granulares, etc., [24]. Existen algunos estudios para medir la conductividad térmica efectiva de estos, aunque, en general, presentan limitaciones con la continuidad de las fases de un material a otro; por lo que incluso se pueden presentar fases adicionales [17]. Asimismo, la geometría de los mismos es un inconveniente debido a que, en la práctica, no todas las partículas tienen la misma forma y tamaño; variando considerablemente el factor de forma, que se toma en cuenta en algunas fórmulas para el cálculo de propiedades de los compuestos [17], [18], [24], [26]. Por lo tanto, los métodos matemáticos de la física son una alternativa necesaria a tener en cuenta para determinar las propiedades efectivas de materiales compuestos.

El presente trabajo tiene como objetivo calcular numéricamente la conductividad y difusividad térmicas efectivas en compuestos sólidos heterogéneos, mediante la simulación computacional a través del análisis por elementos finitos; considerando que, el mecanismo de propagación de calor solo tiene lugar por conducción. Se emplea un método no estacionario que aprovecha la radiación luminosa como fuente externa de calor y constante que incide sobre uno de los extremos $(x=L)$ de una barra térmicamente aislada, en el otro extremo $(x=0)$; la temperatura se mantiene constante (entre 10 y $20{ }^{\circ} \mathrm{C}$ ). El flujo de calor se mide en régimen no estacionario por lo que posee la ventaja de ser una medición relativamente rápida y con un mínimo aumento de temperatura; de esta manera, se evita las pérdidas por radiación. Asimismo, para calcular las propiedades térmicas no se necesita conocer ni las características térmicas ni geométricas de los materiales que integran el compuesto, contrario de otros trabajos [17-26].

\section{Materiales y métodos}

\subsection{Metodología de la simulación}

Se estudia, en primer lugar, una barra homogénea de cobre de 0,15 $\mathrm{m}$ de longitud, con una sección cuadrada de lado igual a $0,007 \mathrm{~m}$ como indica la Figura 1. El software utilizado SolidWorks® 2016 (Licencia: 9710 211750729710 M4R6 FH89), muestra la distribución de temperatura para todos los puntos de la barra a partir del calentamiento de uno de los extremos $(x=L)$, en función del tiempo.

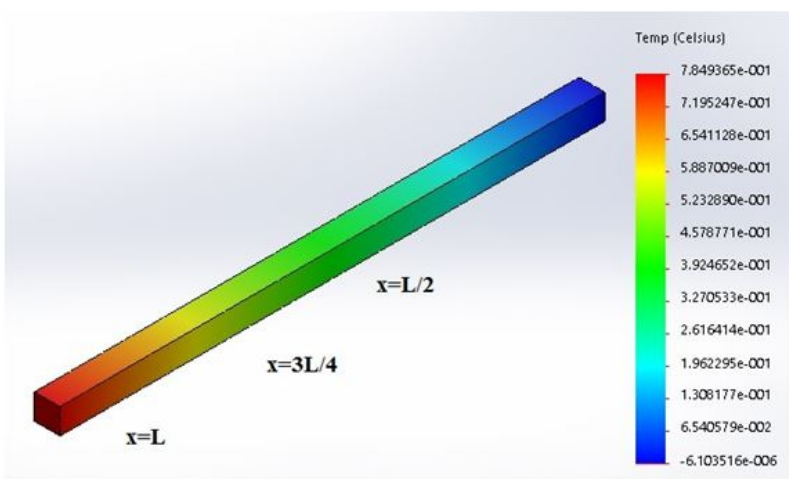

Figura 1. Distribución de la temperatura a lo largo de una barra de cobre de sección cuadrada de longitud $L=0,15$ $m$ y lado $a=0,007 m$. Con estas dimensiones se garantiza las características unidimensionales del sistema propuesto.

Luego de este primer estudio con un material homogéneo conocido se comprueba que los valores de conductividad $(\chi)$ y difusividad $(\alpha)$ térmicas se ajustan satisfactoriamente [27].

En la segunda etapa, se reproduce la simulación, esta vez se obtiene la distribución de la temperatura a lo largo de una barra de cobre de sección cuadrada con cuatro inserciones de plomo de 0,003 $\mathrm{m}$ de longitud y 2,1.10-5 $\mathrm{m}^{2}$ de área de sección transversal, de tal forma que se obtiene un compuesto de cobre $(65,7 \%)$-plomo $(34,3 \%)$, como indica la Figura 2.

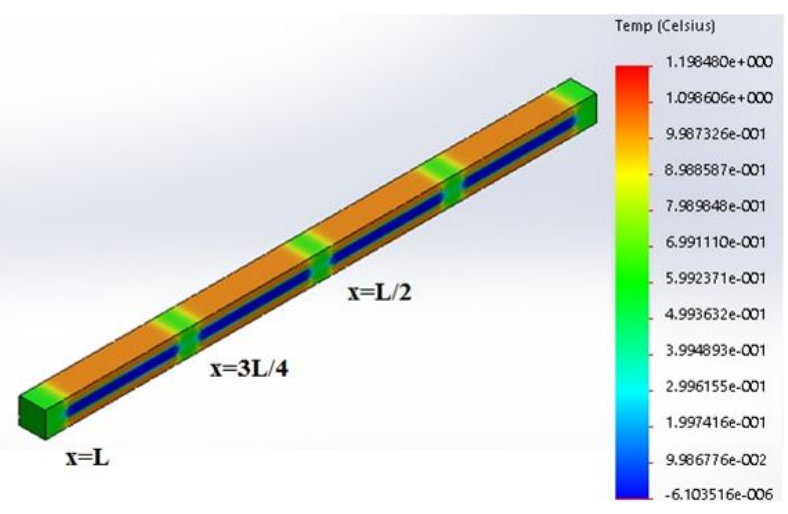

Figura 2. Distribución de la temperatura a lo largo de un material compuesto de cobre (65,7 \%)-plomo $(34,3 \%)$ en forma de barra de longitud $L=0,15 \mathrm{~m}$ y lado $a=0,007 \mathrm{~m}$; con cuatro inserciones de plomo de $0,003 \mathrm{~m}$ de longitud y 2,1·10-5 $\mathrm{m}^{2}$ de área de sección transversal.

En las Figuras 1 y 2, los valores de temperaturas que se muestran en la distribución sobre cada punto de las barras son las temperaturas alcanzadas en estado estacionario: $\Delta T_{e s}$. Sin embargo, para calcular la 
conductividad y difusividad térmicas bajo este método no se necesita llegar a ese valor, como se describe a continuación.

\subsection{Aspectos teóricos}

En el estudio de la propagación del calor en una barra sólida se tiene en cuenta solo el proceso de conducción a lo largo de la misma; las pérdidas de calor por convección y radiación deben ser minimizadas. En este sentido, gracias a un adecuado diseño experimental se logra este propósito. A saber: 1) el poco aumento de la temperatura $\Delta T$ evita pérdidas por radiación, 2) por ser una barra sólida no se aprecia la convección; de forma tal que se puede aplicar la ecuación parabólica del calor para conocer la distribución de temperaturas en toda la barra.

$$
\left\{\begin{array}{l}
\frac{\partial T}{\partial t}(x, t)=\alpha \frac{\partial^{2} T}{\partial x^{2}}(x, t) \\
T(x, 0)=T_{0} \\
T(0, t)=T_{0} \\
\frac{\partial T(L, t)}{\partial x}=Q
\end{array} \quad\{\forall 0<x<L, t<0\}\right.
$$

donde:

$\alpha$ es la difusividad térmica

$Q$ es el flujo de calor constante a través del extremo $x=L$.

Homogeneizando las condiciones de frontera, teniendo en cuenta el método de separación de variables, se obtiene el incremento de la temperatura como función del tiempo $t$ y la coordenada $x$; cuya solución es [28]:

$$
\begin{array}{r}
\Delta T(x, t)=p_{0}\left\{\frac{x}{L}-\frac{8}{\pi^{2}} \sum_{n=0}^{\infty} \frac{(-1)^{n} \cdot e^{-\left(p_{n} \cdot t\right)}}{(2 n+1)^{2}} .\right. \\
\left.\cdot \operatorname{sen}\left[(2 n+1) \frac{\pi x}{2 L}\right]\right\}
\end{array}
$$

donde:

$p_{0}=\frac{q L}{\chi^{A}}$, que representa la variación de temperatura alcanzada en el estado estacionario, $q$ es el calor, $L$ la longitud de la barra, $A$ el área de la sección transversal, $\chi$ la conductividad térmica y $p n=p n(\alpha, n)$, es función de la difusividad térmica y de los términos dentro de la serie. Está asociado con el tiempo que se demora el sistema en alcanzar el estado estacionario e igualmente con la frecuencia con que oscilan los valores de temperatura en cada punto de la barra mientras avanza el tiempo.

Utilizando el método de los mínimos cuadrados se ajustan los puntos de la simulación a la ecuación (2) mediante el programa Mathematica ${ }^{\circledR} 11.0$ (Licencia: 3657-5751-TEGG73). El número de términos de la sumatoria se calcula aproximadamente, de manera que en $x=L$ y $t=0$ la variación de temperatura que se aprecie por el sensor que mide sea igual a cero, o sea: $\Delta T(l, 0)=0$. A partir de los valores obtenidos para $p_{0}$ y $p_{n}$ se determinan los correspondientes de conductividad y difusividad térmicas.

\section{Resultados y discusión}

\subsection{Barra de cobre}

Se muestran los resultados de la primera etapa en donde se trabajó con la barra de cobre. Se puede apreciar en la Figura 3, una convergencia absoluta entre la solución teórica de la ecuación (2) y los datos arrojados por la simulación para los diferentes valores de la coordenada $x$, en que fue monitoreado.

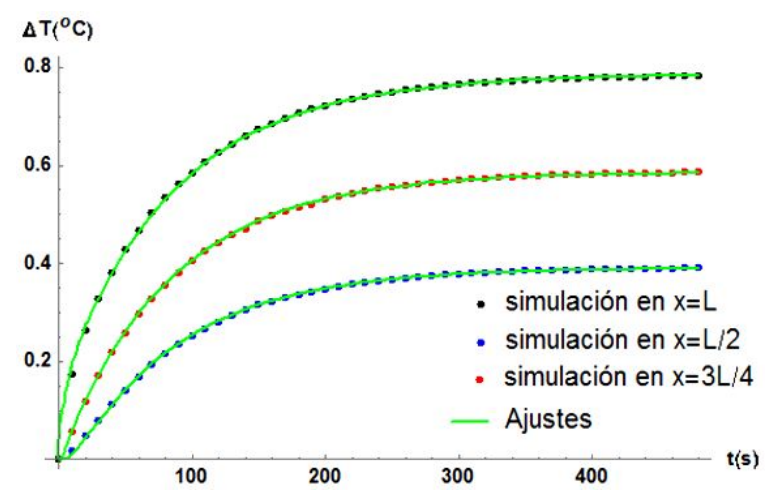

Figura 3. Gráfico del ajuste de la distribución de temperatura vs. tiempo cuando se calienta la barra de cobre en la posición $(x=L)$. Se tiene una convergencia absoluta entre simulación y modelo en los tres puntos de monitoreo.

De tal forma se obtuvo los valores de conductividad y difusividad térmicas presentados en la Tabla $1 \mathrm{y}$, como se aprecia, la coincidencia entre los valores para distintas posiciones es notoria. Esto indica que, obviamente, las características térmicas no dependen de la coordenada si hablamos de un material homogéneo. Asimismo, los valores calculados de conductividad y difusividad térmicas se aproximan significativamente a los ya reportados, con un error relativo promedio de solo $0,01 \%$ y $0,7 \%$ en la conductividad y difusividad térmicas respectivamente [27].

Tabla 1. Comparación entre los valores de conductividad $\chi\left[\mathrm{W} / \mathrm{m}^{\circ} \mathrm{C}\right]$ y difusividad $\alpha \cdot 10^{-6}\left[\mathrm{~m} / \mathrm{s}^{2}\right]$ térmicas reportados y los calculados en tres puntos diferentes de una barra de cobre homogénea.

\begin{tabular}{llcl}
\hline $\begin{array}{l}\text { Valores } \\
\text { reportados }\end{array}$ & \multicolumn{3}{l}{$\begin{array}{l}\text { Valores de conductividad } \\
\text { dad térmicas calculados }\end{array}$} \\
\hline & $(\mathbf{x}=\mathbf{L})$ & $(\mathbf{x}=\mathbf{3 L} / \mathbf{4})$ & $(\mathbf{x}=\mathbf{L} / \mathbf{2})$ \\
\hline$\chi_{\text {cobre }}=390,0$ & $\chi=389,7$ & $\chi=390.0$ & $\chi=390.2$ \\
$\alpha_{\text {acobre }}=112,4$ & $\alpha=105,1$ & $\alpha=105,8$ & $\alpha=106,6$ \\
\hline
\end{tabular}




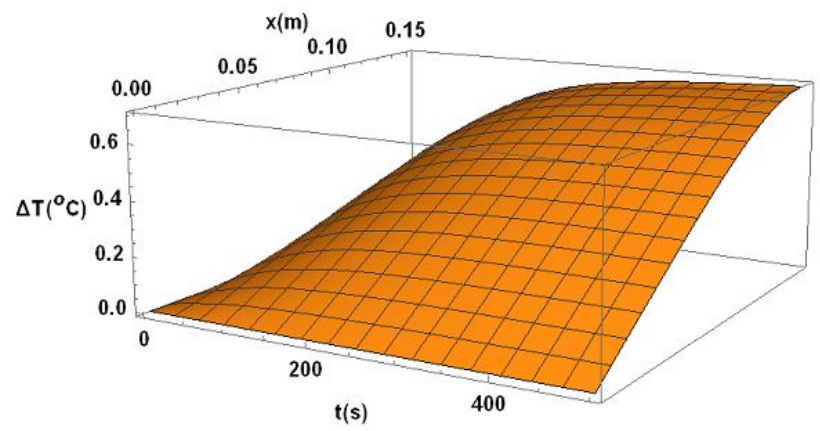

Figura 4. Gráfico de la variación de la temperatura en función del tiempo $t$ y la coordenada $x$, para la barra de cobre homogénea: $q=0,1 \mathrm{~W} ; A=4,9 \cdot 10^{-5} \mathrm{~m}^{2}$; $L=0,15 \mathrm{~m}$.

En la Figura 4 se aprecia el gráfico de la distribución de temperatura en función de la coordenada $x$ y el tiempo $t$, según la ecuación (2), con los valores de $\chi$ y $\alpha$ obtenidos en la Tabla $1, q=0,1 W, A=4,9.10^{-5} \mathrm{~m}^{2}$, $L=0,15 \mathrm{~m}$. Nótese que, el aumento de temperatura es pequeño (menor a un grado) lo que resulta conveniente a la hora de poner en marcha esta medición en el laboratorio.

\subsection{Compuesto}

A continuación, en la Figura 5 se muestra los resultados de la segunda etapa, donde se trabajó con un compuesto como indica la Figura 2.

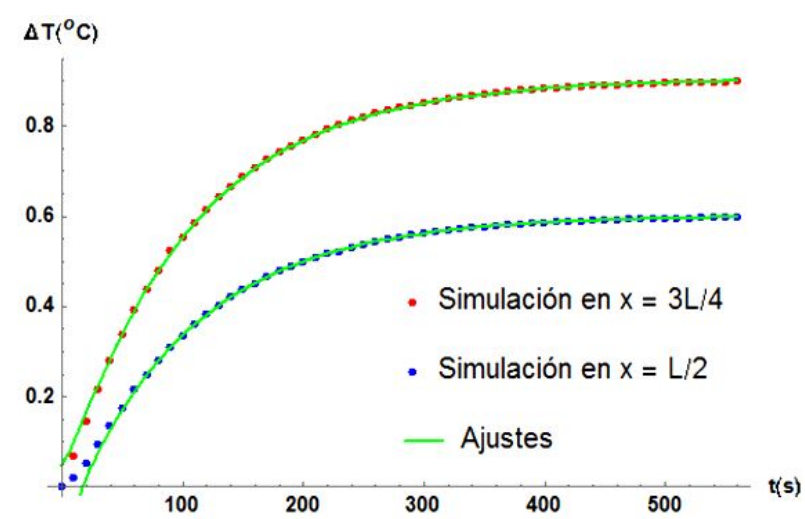

Figura 5. Gráfico del ajuste de la distribución de temperatura vs. tiempo cuando se calienta el compuesto (cobre $65,7 \%$ - plomo $34,3 \%$ ) en la posición $x=L$. Se tiene una convergencia absoluta después de los primeros $30 \mathrm{~s}$.

Se debe acotar que, en esta segunda etapa se monitoreó la variación de temperatura en las posiciones $x=3 L / 4$ y $x=L / 2$, debido a que están inmersas dentro del compuesto. Igualmente, en la Figura 5 se observa que el modelo comienza a ajustarse absolutamente después de los primeros 30 segundos a partir de que comienza a recibir el calor por el extremo $x=L$. Esta discrepancia puede estar asociada a la baja conductividad térmica del plomo de forma tal que, en las posiciones $x=3 L / 4$ y $x=L / 2$, aún no llega un flujo de calor significativo al comienzo; por lo que los datos resultan un poco dispersos, esto se puede solucionar aumentando la potencia de $q$ en $x=L$.

Tabla 2. Valores de conductividad $\chi\left[\mathrm{W} / \mathrm{m}{ }^{\circ} \mathrm{C}\right]$ y difusividad $\alpha \cdot 10^{-6}\left[\mathrm{~m} / \mathrm{s}^{2}\right]$ térmicas efectivas calculados en dos puntos diferentes del compuesto.

\begin{tabular}{cc}
\hline$(\mathrm{x}=3 \mathbf{L} / 4)$ & $(\mathrm{x}=\mathbf{L} / \mathbf{2})$ \\
$\chi=254.0$ & $\chi=254.6$ \\
$\alpha=86,6$ & $\alpha=87,5$ \\
\hline
\end{tabular}

En la Tabla 2 aparecen los resultados de la conductividad y difusividad térmicas efectivas calculadas para el compuesto. Como se aprecia, los valores efectivos tanto de $\chi$ como de $\alpha$ se encuentran en el intervalo entre los valores del plomo y cobre, por lo que, el resultado es lógico y adecuado. En segundo lugar, se nota precisión (si bien no significa total exactitud) en los resultados pues, ambos puntos de monitoreo, arrojan cifras muy semejantes; convirtiéndolo en un método consistente.

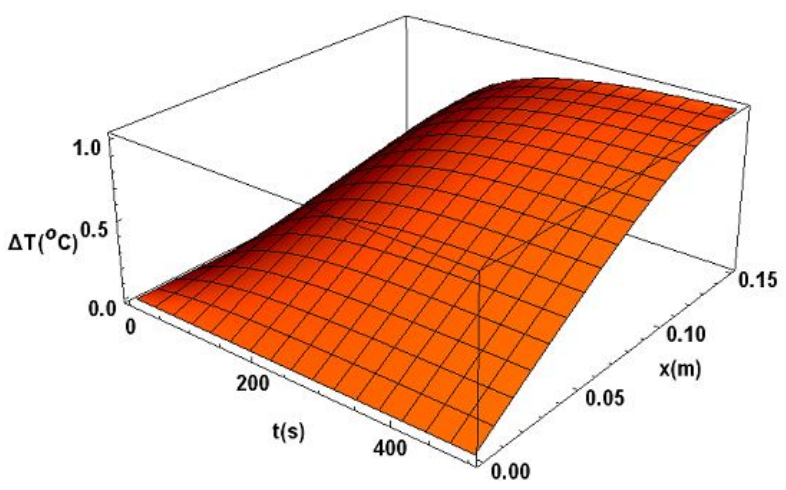

Figura 6. Gráfico de la variación de la temperatura en función del tiempo $t$ y la coordenada $x$, en el compuesto: $q=0,1 W ; A=4,9 \cdot 10^{-5} \mathrm{~m}^{2} ; L=0,15 \mathrm{~m}$.

En la Figura 6 se aprecia el gráfico de la distribución de temperatura en función de la coordenada $x$ y el tiempo $t$, según la ecuación 2 , con los valores de $\chi$ y $\alpha$ efectivos obtenidos en la Tabla $2, q=0,1 \mathrm{~W}$, $A=4,9 \cdot 10^{-5} \mathrm{~m}^{2}, L=0,15 \mathrm{~m}$. Nótese que, el aumento de temperatura continúa siendo pequeño (alrededor de un grado), sin embargo, mayor que en el caso de la barra de cobre homogénea. Esto se debe a que, al ser la conductividad térmica efectiva del compuesto menor a la del cobre, la velocidad de propagación del flujo de calor es menor; por lo tanto, se acumula más calor en los puntos monitoreados.

\subsection{Implementación del método}

Hasta aquí, se ha calculado numéricamente la conductividad y difusividad térmicas efectiva de un compuesto 
a base de cobre $(65,7 \%)$ - plomo $(34,3 \%)$. Si bien es necesario ampliar el número de simulaciones a otros tipos de configuraciones y, de ser posible, llevarlo a la práctica, se logra estimar bajo condiciones adecuadas los valores térmicos efectivos, que se encuentran en la Tabla 2 .

En este sentido, se ha utilizado una herramienta informática SolidWorks®2016, cuyo método de cálculo de elementos finitos, está validado para la obtención de propiedades térmicas en un compuesto unidimensional, para las condiciones en que se diseñó la muestra [29]. Asimismo, los valores de la conductividad y difusividad térmicas efectivas obtenidas están dentro del intervalo que se establece en [30].

\section{Conclusiones}

La metodología numérica presentada en este trabajo se puede convertir en una herramienta para el cálculo de la conductividad y difusividad térmicas efectivas de un compuesto sólido unidimensional. Como ventaja, no se necesita, en general, conocer ni la geometría ni las dimensiones exactas del material insertado (aunque este debería estar disperso), tampoco las propiedades térmicas de los materiales constituyentes, pues solo se necesita monitorear la temperatura en varios puntos y ajustar los valores de $\chi$ y $\alpha$ a la ecuación 2 .

\section{Referencias}

[1] W. J. Parker, R. J. Jenkins, C. P. Butler, and G. L. Abbott, "Flash method of determining thermal diffusivity, heat capacity, and thermal conductivity," Journal of Applied Physics, vol. 32, no. 9, pp. 1679-1684, 1961. [Online]. Available: http://dx.doi.org/10.1063/1.1728417

[2] A. Bouguerra, A. Aït-Mokhtar, O. Amiri, and M. Diop, "Measurement of thermal conductivity, thermal diffusivity and heat capacity of highly porous building materials using transient plane source technique," International Communications in Heat and Mass Transfer, vol. 28, no. 8, pp. 1065-1078, 2001. [Online]. Available: http: //dx.doi.org/10.1016/S0735-1933(01)00310-4

[3] J. K. Carson, S. J. Lovatt, D. J. Tanner, and A. C. Cleland, "Thermal conductivity bounds for isotropic, porous materials," International Journal of Heat and Mass Transfer, vol. 48, no. 11, pp. 2150-2158, 2005. [Online]. Available: http://dx.doi.org/10.1016/j. ijheatmasstransfer.2004.12.032

[4] J. Hone, M. Whitney, C. Piskoti, and A. Zettl, "Thermal conductivity of single-walled carbon nanotubes," Physical Review B: Condensed Matter and Materials Physics, vol. 59, pp. R2514-R2516, Jan 1999. [Online]. Available: https://doi.org/10.1103/PhysRevB.59.R2514

[5] J. A. P. Lima, E. Marín, O. Correa, M. G. da Silva, S. L. Cardoso, C. Gatts, C. E. Rezende, H. Vargas, and L. C. M. Miranda, "Measurement of the thermal properties of liquids using a thermal wave interferometer," Measurement Science and Technology, vol. 11, no. 10, pp. 1522-1526, 2000. [Online]. Available: https://goo.gl/owQmKY

[6] L. Lira-Cortés, O. J. González, and E. MéndezLango, "Medición de la conductividad térmica de algunos materiales utilizados en edificaciones," in Simposio de Metrología 2008, México, 2008. [Online]. Available: https://goo.gl/MGCFWM

[7] L. Lira-Cortés, S. García, E. Méndez-Lango, and E. González, "Conductividad térmica en materiales," in Simposio de Metrología 2010, México, 2010. [Online]. Available: https://goo.gl/pc5xZh

[8] A. Corona and G. Martínez, "Conducción térmica en una varilla de cobre," Latin-American Physics Education Network, vol. 5, no. 4, pp. 820-823, 2011. [Online]. Available: https://goo.gl/SjKZz9

[9] J. A. Gutiérrez and A. D. González, "Determinación experimental de conductividad térmica de materiales aislantes naturales y de reciclado," Avances en Energías Renovables y Medio Ambiente, vol. 16, pp. 08.41-08.48, 2012. [Online]. Available: https://goo.gl/zRREW4

[10] M. E. González, A. Denis, and A. Soba, "Modelización de la conductividad térmica del uo2 y (u,gd)o2 bajo irradiación. implementación en el código dionisio," ANALES AFA, vol. 25, no. 4, pp. 211-213, 2014. [Online]. Available: https://goo.gl/86mMTe

[11] A. R. Warrier, R. Jayakrishnan, T. T. John, C. S. Kartha, and K. P. Vijayakumar, "Study on optical, electronic and thermal properties of $\beta$-in2s3 thin films using photothermal beam deflection technique," Journal of Materials Science: Materials in Electronics, vol. 27, no. 4, pp. 3628-3636, 2016. [Online]. Available: http://dx.doi.org/10.1007/s10854-015-4201-y

[12] K. Martínez, E. Marín, C. Glorieux, A. LaraBernal, A. Calderón, G. P. na Rodríguez, and R. Ivanov, "Thermal diffusivity measurements in solids by photothermal infrared radiometry: Influence of convection-radiation heat losses," International Journal of Thermal Sciences, vol. 98, pp. 202-207, 2015. [Online]. Available: http: //dx.doi.org/10.1016/j.ijthermalsci.2015.07.019 
[13] L. Velasco, L. Goyos, R. Delgado, and L. Freire, "Instalación para medición de conductividad térmica en composites basados en residuos de biomasa," Enfoque UTE, vol. 7, no. 2, pp. 69-81, 2016. [Online]. Available: https://goo.gl/BUbGpB

[14] N. Cobîrzan, A.-A. Balog, B. Belean, G. Borodi, D. Dadârlat, and M. Streza, "Thermophysical properties of masonry units: Accurate characterization by means of photothermal techniques and relationship to porosity and mineral composition," Construction and Building Materials, vol. 105, pp. 297-306, 2016. [Online]. Available: http: //dx.doi.org/10.1016/j.conbuildmat.2015.12.056

[15] J. A. Ibáñez, F. J. Abellán, R. P. Valerdi, and J. A. García, "Conductividad térmica de una barra de cobre. estudio experimental del transitorio," Latin-American Journal of Physics Education, vol. 2, no. 3, pp. 259-267, 2008. [Online]. Available: https://goo.gl/y1NCAF

[16] S. E. Gustafsson, "Transient plane source techniques for thermal conductivity and thermal diffusivity measurements of solid materials," Review of Scientific Instruments, vol. 62, no. 3, pp. 797-804, 1991. [Online]. Available: http://dx.doi.org/10.1063/1.1142087

[17] R. L. Hamilton and O. K. Crosser, "Thermal conductivity of heterogeneous two-component systems," Industrial \& Engineering Chemistry Fundamentals, vol. 1, no. 3, pp. 187-191, 1962. [Online]. Available: http://dx.doi.org/10.1021/i160003a005

[18] J. C. Maxwell, A treatise on Electricity and Magnetism, 2nd ed., 1881, vol. 1, p. 435. [Online]. Available: https://goo.gl/a3caJn

[19] L. Sassi, F. Mzali, A. Jemnia, and S. B. Nasrallah, "Hot-wire method for measuring effective thermal conductivity of porous media," Journal of Porous Media, vol. 8, pp. 97-113, 2005. [Online]. Available: http://dx.doi.org/10.1615/JPorMedia.v8.i2.10

[20] G. Peña, J. Moreno, and E. Vera, "Conductividad térmica efectiva promedio de polvos de arcillas utilizadas en la industria cerámica del área metropolitana de San José de Cúcuta," Revista Colombiana de Física, vol. 40, pp. 278-280, 2008. [Online]. Available: https://goo.gl/62FRHg

[21] N. Wakao and K. Kato, "Effective thermal conductivity of packed beds," Journal of Chemical Engineering of Japan, vol. 2, pp. 24-33, 1969. [Online]. Available: https://goo.gl/azdxgp

[22] D. Shonnard and S. Whitaker, "The effective thermal conductivity for a pointcontact porous medium: an experimental study," International Journal of Heat and Mass Transfer, vol. 32, no. 3, pp. 503-512, 1989. [Online]. Available: http: //dx.doi.org/10.1016/0017-9310(89)90138-5

[23] N. S. Basheer, B. R. Kumar, A. Kurian, and S. D. George, "Thermal conductivity measurement of organic solvents incorporated with silver nanoparticle using photothermal techniques," IOP Conference Series: Materials Science and Engineering, vol. 73, no. 1, p. 012039, 2015. [Online]. Available: http: //dx.doi.org/10.1088/1757-899X/73/1/012039

[24] J. Bravo, R. Guinovart, G. López, R. Rodríguez, and F. J. Sabina, "Acerca de la homogeneización y propiedades efectivas de la ecuación del calor," Revista Visión Electrónica, vol. 7, no. 1, pp. 149-159, 2013. [Online]. Available: https://goo.gl/KTpdXu

[25] S. Nie and C. Basaran, "A micromechanical model for effective elastic properties of particulate composites with imperfect interfacial bonds," International Journal of Solids and Structures, vol. 42, no. 14, pp. 4179-4191, 2005. [Online]. Available: http://dx.doi.org/10.1016/j.ijsolstr.2004.12.009

[26] G. Peña, A. Calderón, R. A. Muñoz, A. Florido, O. Flores, and C. Falcony, "Influencia del tamańo de grano en la conductividad térmica a altas temperaturas en polvos aislantes de $\mathrm{MgO}$," Superficies y vacio, vol. 14, pp. 44-48, 2002. [Online]. Available: https://goo.gl/gUQ7fi

[27] W. Benenson, J. W. Harris, H. Stöcker, and H. Lutz, Handbook of physics, 1st ed. Springer-Verlag, New York, 2002, vol. 2, pp. 788-795. [Online]. Available: https: //dx.doi.org/10.1007/0-387-21632-4

[28] R. Hechavarría, O. Delgado, and F. Pazmiño, "Estudio de un nuevo procedimiento para medir la conductividad y difusividad térmicas de materiales," INGENIUS, no. 17, pp. 15-22, 2017. [Online]. Available: http: //dx.doi.org/10.17163/ings.n17.2017.02

[29] M. A. E. C. Rodrigo P. A. Rocha, "Computation of the effective conductivity of unidirectional fibrous composites with an interfacial thermal resistance," Numerical Heat Transfer, Part A: Applications, vol. 39, no. 2, pp. 179-203, 2001. [Online]. Available: http://dx.doi.org/10.1080/10407780118981

[30] G. López, J. Bravo, M. E. Cruz, R. Guinovart, and R. Rodríguez, "Cotas variacionales para coeficientes efectivos en compuestos con contacto imperfecto," Revista Visión Electrónica, vol. 7, no. 1, pp. 53-64, 2013. [Online]. Available: https://goo.gl/8nxXzc 